\title{
Patrones reproductivos del lobo marino común, Otaria flavescens (Shaw 1800), en el centro-sur de Chile
}

\author{
Breeding patterns in southern sea lions, Otaria flavescens (Shaw 1800), \\ in south-central Chile \\ HÉCTOR J. PAVÉS ${ }^{1}$, ROBERTO P. SCHLATTER ${ }^{1}$ \& CRISTIAN I. ESPINOZA ${ }^{2}$ \\ ${ }^{1}$ Instituto de Zoología, Universidad Austral de Chile, Casilla 567, Valdivia, Chile \\ ${ }^{2}$ Servicio Nacional de Pesca, Melinka, Chile; \\ e-mail para correspondencia: hpaves@ surnet.cl
}

\begin{abstract}
RESUMEN
El desfase latitudinal de los eventos reproductivos en Otaria flavescens sugiere la existencia de una adaptación fisiológica y conductual a condiciones ambientales locales. La identificación de cambios en los patrones conductuales intraespecíficos frente a modificaciones ambientales sugieren una relación entre estos. Se pretende revisar los patrones reproductivos del O. flavescens, determinando la existencia de una sincronización y estabilidad en ellos durante las temporadas 1996-1997 y 1997-1998 en la colonia de Punta Lobería, Chile. Se realizaron observaciones directas de las agrupaciones y conductas reproductivas empleando binoculares, y apoyadas por el uso de material fílmico y fotográfico. Los resultados evidenciaron modificaciones en, (a) el número de harenes (de 28 disminuye a 19), (b) la distribución espacial de los harenes (70 \% de los harenes durante 1997-1998 se establecieron en el lado norte de la playa reproductiva, durante la temporada anterior estos se distribuyeron a lo largo de toda la playa), (c) proporción etaria (un macho: 12 hembras: un juvenil: siete cachorros, desciende a un macho: nueve hembras: dos juveniles: seis cachorros), (d) índice de poliginia (de 12,4 a 7,8 HPMT y de 11 a 9 HP), y (e) la población organizada en harenes (disminución del 10,3\%). Estas variaciones estarían relacionadas con la reducción del espacio óptimo para el establecimiento de harenes debido a la incorporación de suelo/roca posterior a un derrumbe invernal. Los patrones conductuales reproductivos que se mantuvieron invariables durante las temporadas fueron, (a) la estacionalidad de los harenes (constitución durante la cuarta semana de diciembre y disolución en la cuarta semana de febrero), (b) el período de partos (inicio durante la segunda semana de enero, finalización durante la cuarta semana de enero), y (c) el período de cópulas (inicio durante la tercera semana de enero, finalización durante la segunda semana de febrero). Por lo tanto, O. flavescens presentaría conductas modificables y permanentes que se despliegan según las condiciones ambientales. Donde las conductas reproductivas modificadas potencialmente mitigarían pérdidas en aspectos reproductivos, y las conductas reproductivas permanentes, estarían relacionadas con procesos fisiológicos y conductuales seleccionados bajo las condiciones ambientales. Estos patrones reproductivos al ser comparados con las de otras localidades son congruente con un efecto latitudinal, debido a la variación registrada tanto en la estacionalidad de ellos como en la duración de los mismos (e.g., período de partos y cópulas).
\end{abstract}

Palabra clave: Otaria flavescens, patrones reproductivos, sincronización reproductiva, poliginia.

\begin{abstract}
The latitudinal effect of reproductive patterns for Otaria flavescens suggests the existence of behavioral and physiological adaptation to local environmental conditions. The identification of intraspecific behavioral pattern changes due to these environmental conditions suggest a relation among them. We pretend here to determine reproductive synchronization and reproductive stability of $O$. flavescens in the Punta Lobería colony during two breeding seasons 1996-1997 and 1997-1998. Direct observations of groups and breeding behavior were watched with binoculars backed by video and photography. Results evidenced modification in (a) the number of harems (decreased from 28 to 19 during first to the second seasons), (b) the spatial distribution of harems (70\% of these were located in northern part of the reproductive beach during the 1997-1998 season whereas they occupied the whole beach rather uniformly during the previous season), (c) age structure (one male: 12 females: one juvenile and seven pups decreased to one male: nine females: two juveniles and six pups), (d) the polygyny index (decreased from 12.4 to 7.8 HPMT and from 11 to 9 HP), and (e) the reproductive population organized in harems (decreased of up to $10.3 \%$ ). These variations would be related to optimum space reduction for harem establishment after an earth/rock slide over the area. Behavioral reproductive patterns remaining stable during
\end{abstract}


both seasons were (a) seasonality of harems (harem formation during the fourth week in December, dissolution during the fourth week of February), (b) birth period (beginning during the second week of January and ending during the fourth week of January), and (c) mating period (beginning during the third week of January, ending during the second week in February). Otaria flavescens exhibited modifiable and stable behavioral patterns according to environmental conditions. Modified breeding behavior potentially would mitigate loss of reproductive output, and stable breeding behavior would be related to physiological and behavioral processes according to environmental conditions. Thus, reproductive patterns when compared to other known reproductive localities in Chile are consistent with a latitudinal effect due to variations registered by their seasonality, as well as its duration (e.g., mating and birth periods).

Key words: Otaria flavescens, reproductive patterns, reproductive synchronization, polygyny index.

\section{INTRODUCCIÓN}

Las actividades reproductivas del lobo marino común, Otaria flavescens (Shaw 1800), al igual que la de otros Otariidae, son llevadas a cabo en lugares específicos (i.e., parideros o colonias reproductivas) y en períodos discretos (i.e., temporadas reproductivas) (Sielfeld 1983, Riedman 1990). En estos parideros, la gran cantidad de ejemplares congregados desarrollan un conjunto de patrones conductuales característicos no manifestados durante el resto del año, e.g., formación de "harenes", pariciones y cópulas (Vaz-Ferreira 1965, Campagna 1985, Sielfeld 1999, Acevedo et al. 2003). El período donde se registran estos patrones son principalmente en los meses primavera-verano, fecha en la cual el aumento de la productividad y las favorables condiciones meteorológicas potencian un ambiente propicio para la sobrevivencia y el desarrollo de la descendencia (Bowen 1991, Majluf 1992, Testa 2002, Trites \& Antonelis 1994).

La periodicidad de las temporadas reproductivas está directamente relacionada con las características del ciclo reproductivo de los otáridos, el cual a su vez es el reflejo de una presión de selección natural sobre la descendencia y los progenitores (Testa 2002). El ciclo reproductivo en estas especies se caracteriza por presentar un retardo en la implantación embrionaria de 2 a 3 meses con un período fijo de gestación de 9 meses (Costa 1991). Este retardo, que en definitiva determinaría la fecha de las pariciones, podría estar relacionado con factores exógenos (e.g., disponibilidad de alimento, condiciones meteorológicas) y/o endógenos (e.g., condiciones fisiológicas de la hembra, fecha implante embrionario) (Riedman 1990, Campagna et al. 1993, Higgins 1993, Trites \& Antonelis 1994, Soto et al. 2004).
A lo largo de toda la zona templada del cono sudamericano es posible identificar ejemplares del lobo marino común (Otaria flavescens), los cuales se distribuyen desde el norte del Perú ( $\left.4^{\circ} \mathrm{S}\right)$ hasta el Cabo de Hornos e isla Diego Ramírez, en el Océano Pacífico. En el Atlántico, estos se encuentran desde Brasil $\left(29^{\circ} \mathrm{S}\right)$ hasta Tierra del Fuego e Islas MalvinasFalklands (Sielfeld 1983, Cappozzo \& Junín 1991, FAO 1993, Sielfeld 1999, Acevedo et al. 2003).

En esta especie, debido a su amplia área de distribución, Campagna (1985) ha mencionado la existencia de un efecto latitudinal sobre los eventos reproductivos entre poblaciones del Atlántico sur, aspecto que ha sido corroborado para otros Otariidae (Riedman 1990, Campagna et al. 1993, Goldsworthy \& Shaughnessy 1994, García-Aguilar \& Aurioles-Gamboa 2003). Este efecto latitudinal se evidencia en una relación negativa entre el grado de sincronización reproductiva, i.e., el período en el cual se registran el $90 \%$ de las pariciones, y la latitud donde se establecen los otáridos (Boness 1991). Aunque existe este efecto, los patrones reproductivos pueden ser identificados en una determinada lobera durante el mismo período de tiempo (Campagna 1985, Majluf 1992, Trites 1992, Goldsworthy \& Shaughnessy 1994, Acevedo et al. 2003, García-Aguilar \& Aurioles-Gamboa 2003). Este aspecto evidenciaría una relación entre ciclo reproductivo de cada especie de Otariidae y las condiciones ambientales locales (Bowen 1991, Costa \& Gales 2003).

La existencia de esta relación entre las condiciones ambientales locales y la biología reproductiva de estos mamíferos marinos sugiere que posibles alteraciones ambientales locales podrían generar cambios tanto en los eventos como en las conductas reproductivas de la especie. De tal modo, que la presencia de 
perturbaciones en el ambiente podrían modificar los patrones y las conductas reproductivas de $O$. flavescens durante temporadas sucesivas. Inserto en esta problemática, la presente contribución tiene por objeto revisar los patrones reproductivos del lobo marino común, analizando la sincronización y estabilidad en ellos durante temporadas reproductivas sucesivas.

\section{MATERIALES Y MÉTODOS}

Las actividades de terreno fueron desarrolladas en la colonia reproductiva del lobo marino común de tipo permanente, localizada en Punta Lobería (38³9' S, 73²9' O), IX Región de La Araucanía, Chile (Fig. 1). Esta colonia está ubicada en la base de un farellón costero fuertemente erosionado de una altura entre 80$120 \mathrm{~m}$ sobre el nivel de mar y de $1 \mathrm{~km}$ de largo orientada en sentido ONO. La topografía y el fuerte oleaje del sector hacen inaccesible el ingreso a la lobera por tierra o mar, pudiendo solo observarse desde la cima del acantilado circundante. Todo el sector está sujeto a fuertes derrumbes del acantilado que modifican constantemente las características topográficas de la lobera.

En la colonia es posible identificar cinco sectores: Roqueríos Principales (área de descanso), Playa Chica (área reproductiva), Peñón Pilolcura (área reproductiva), Roqueríos sur (área reproductiva) y Playa Grande (área reproductiva). De entre estos sectores se seleccionó la Playa Grande como el área de estudio de la presente investigación, por ser aquella que congrega la mayor población reproductiva y concentra el mayor número de cachorros y cópulas de la lobera (Fig. 2).

\section{Metodología de muestreo}

Los muestreos fueron realizados durante dos temporadas reproductivas consecutivas, 19961997 y 1997-1998, completando un total de 1.200 h de observación. Durante la primera temporada se estableció un campamento en forma continua desde el 18 de diciembre de 1996 hasta el 28 de febrero de 1997. En la segunda temporada se realizaron muestreos durante el 22 y 23 de noviembre de 1997, 6, 7, 20 y 21 de diciembre de 1997, 6-8 de enero de 1998; se estableció un campamento desde el 13 de enero de 1998 al 28 de febrero de 1998.

La zona de observación se ubicó en el farellón costero a $80 \mathrm{~m}$ sobre el nivel del mar, el que abarcó visualmente la Playa Grande y todas las otras zonas de la lobera (Fig. 2). Las observaciones se desarrollaron entre las 8:00 y las 20:00 h realizando las siguientes actividades, supeditadas a las condiciones meteorológicas imperantes: (a) identificación y determinación de la estructura etaria de cada harén; se registró la ubicación y las proporciones etarias de cada agrupación socioreproductiva, monitoreando su número, distribución espacial, composición y tamaño en el tiempo. La estructura de los harenes se determinó por medio de tres censos diarios de las distintas clases etarias o funcionales de los lobos marinos basándose en las características morfológicas y conductuales de cada ejemplar descrita por Vaz-Ferreira (1982), Bianco et al. $(1987)^{1}$, Rivera (1990) y Sepúlveda (1999) (i.e., hembras, juveniles, cachorros y machos territoriales). Los censos fueron realizados por dos investigadores tres veces al día (i.e., mañana, mediodía y al atardecer). Estos censos fueron repetidos hasta que la diferencia entre los observadores fuese inferior al $10 \%$. En todo los censos se emplearon un contador manual y binoculares (Samsung 7-15 x 35, Tasco 7 x 10). Para determinar la estructura etaria de los harenes de cada temporada reproductiva, se promedió el número de hembras, juveniles y cachorros de cada harén en las fechas en que estas se mantuvieron relativamente constante, i.e., entre el 15 de enero y el 15 de febrero de cada año. Sin embargo, los análisis estadísticos de estos datos se realizaron empleando la sumatoria de los machos, hembras, juveniles y cachorros de los harenes para cada temporada (Tabla 1).

También se determinó (b) el nivel de poliginia de los harenes (i.e., cantidad de hembras asociada a un macho). Para ello, se empleó el índice de hembras por macho territorial (HPMT), que describe el número de hembras totales en relación a los machos

\footnotetext{
${ }^{1}$ BIANCO J, A PONCE DE LEÓN \& R VAZ-FERREIRA (1987) Interacciones entre machos y cachorros de Otaria flavescens (Shaw), león marino sudamericano, en el Uruguay. (Pinnipedia, Otariidae). Anais da $2^{\circ}$ Reunião de Trabalho de Especialistas em Mamíferos Aquáticos da América do Sul, Rio Janeiro, Brasil: 24-27.
} 


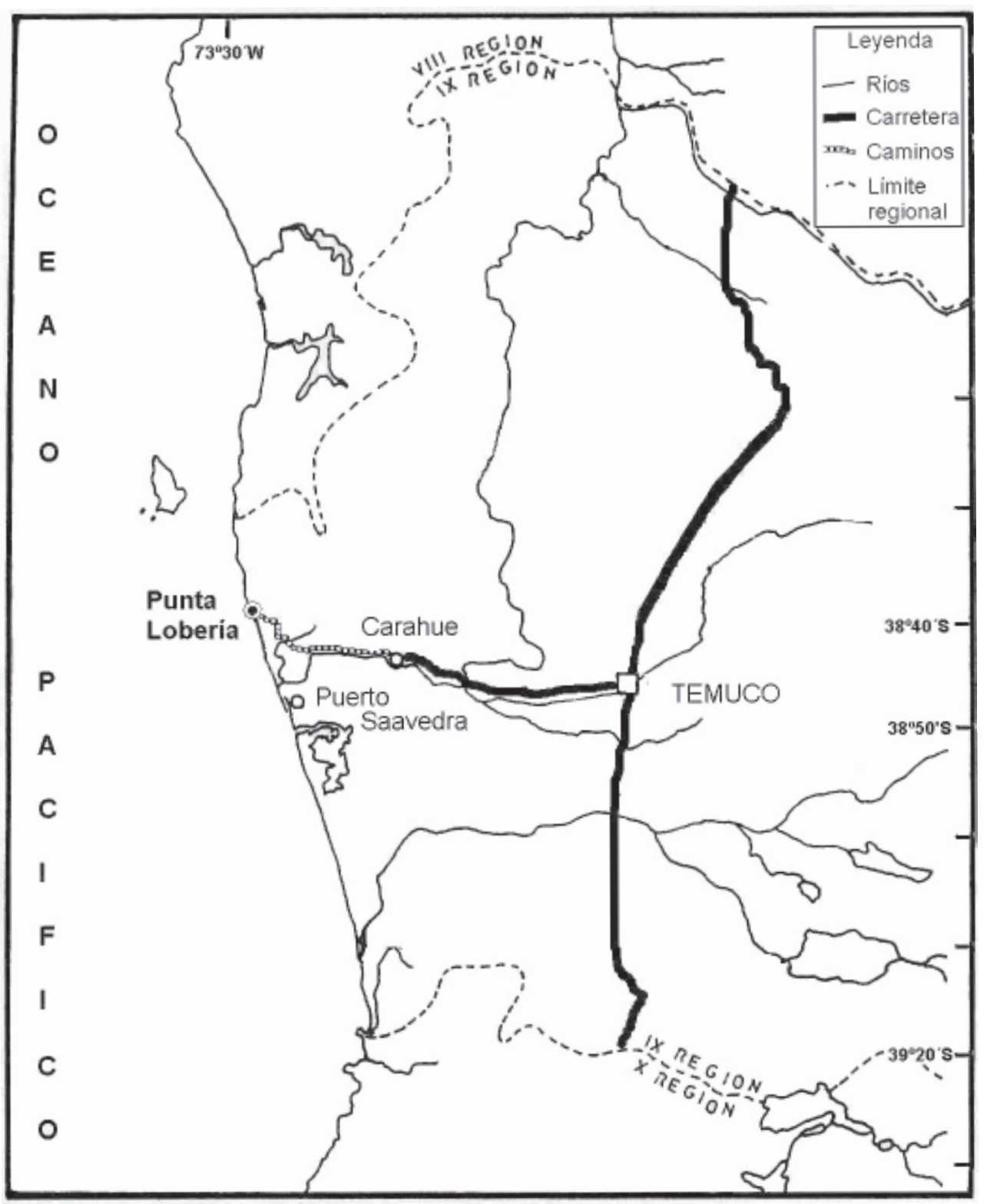

Fig. 1: Ubicación geográfica de Punta Lobería (38³9’ S, $73^{\circ} 29^{\prime}$ O) en la zona costera de la IX Región de Chile.

Geographical location of Punta Lobería (38 $39^{\prime}$ S, $73^{\circ} 29^{\prime}$ W) on the coast of IXth Region of Chile. 

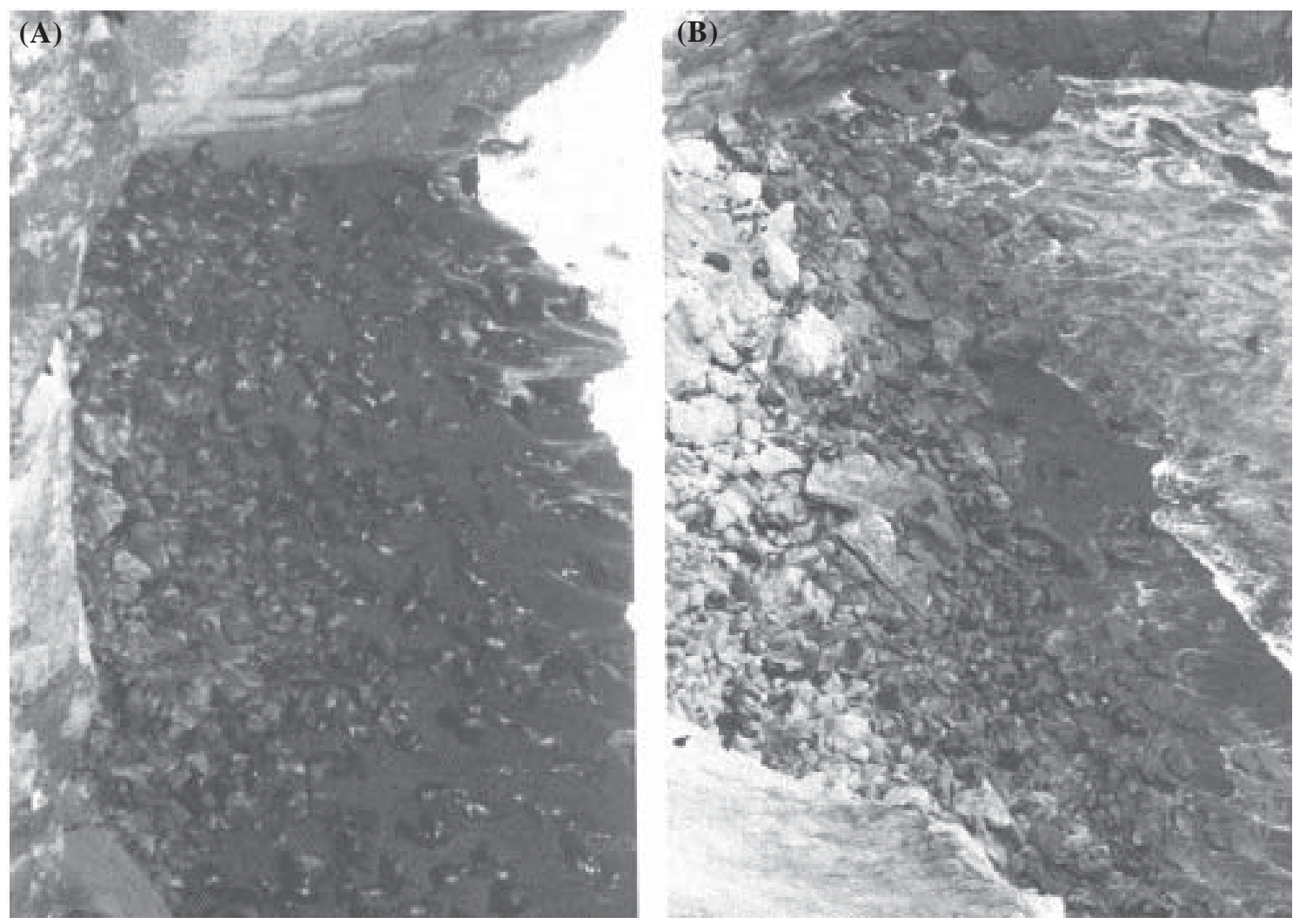

Fig. 2: Fotografía del sector Playa Grande de Punta Lobería durante (A) temporada 1996-1997 y (B) temporada 1997-1998. La playa de arena de suave pendiente en (A) es sustituida por una playa de bolones de arenisca en (B), posterior a un derrumbe del acantilado. (Foto: J. Oporto; H. Pavés).

Image of Playa Grande beach, Punta Lobería during (A) the 1996-1997 season, and (B) the 1997-1998 season. A beach with gentle slope in (A) is replaced by a sand bouldered situation in (B), after a cliff slide. (Photo: J. Oporto; H. Pavés).

\section{TABLA 1}

Variación de la estructura de los harenes durante la temporada reproductiva 1996-1997 y 1997-1998. Se incluye el número de las distintas clases etarias por harén y la cantidad de harenes establecido en la Playa Grande de Punta Lobería, IX Región. Chile

Structure variation for harems during breeding seasons 1996-1997 and 1997-1998. Number of different age class per harem and number of established harems are also given for Punta Lobería, IXth Region, Chile

\begin{tabular}{|c|c|c|c|c|c|c|c|c|c|c|c|c|c|}
\hline & \multicolumn{5}{|c|}{ Temporada 1996-1997 } & & \multicolumn{7}{|c|}{ Temporada 1997-1998 } \\
\hline & 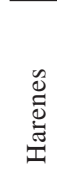 & 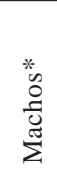 & 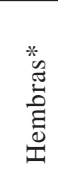 & 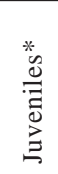 & 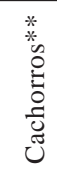 & & 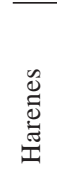 & $\begin{array}{l}{ }^{*} \\
\text { o } \\
\frac{0}{0} \\
\tilde{J} \\
\Sigma\end{array}$ & 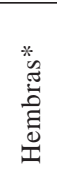 & 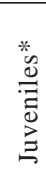 & $\begin{array}{l}* \\
* \\
0 \\
0 \\
0 \\
0 \\
0 \\
0 \\
\tilde{U}\end{array}$ & $\begin{array}{l}0 \\
0 \\
0 \\
\frac{0}{\pi} \\
\frac{\pi}{J}\end{array}$ & 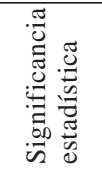 \\
\hline- & - & - & - & - & - & $22 / 11 / 1997$ & 1 & 1 & 18 & 0 & 0 & - & - \\
\hline $26 / 12 / 1996$ & 11 & 1 & 84 & 3 & 0 & $21 / 12 / 1997$ & 1 & 1 & 55 & 27 & 0 & 28.034 & $\mathrm{P}<0,05$ \\
\hline $21 / 01 / 1997$ & 20 & 1 & 12 & 2 & 3 & $19 / 01 / 1998$ & 10 & 1 & 9 & 3 & 4 & 0.746 & $\mathrm{P}>0,05$ \\
\hline $02 / 02 / 1997$ & 28 & 1 & 9 & 1 & 9 & $02 / 02 / 1998$ & 19 & 1 & 10 & 1 & 6 & 0.551 & $\mathrm{P}>0,05$ \\
\hline $09 / 02 / 1997$ & 28 & 1 & 14 & 1 & 9 & 09/02/1998 & 18 & 1 & 9 & 1 & 7 & 0.201 & $\mathrm{P}>0,05$ \\
\hline $23 / 02 / 1997$ & 6 & 1 & 65 & 10 & 42 & $23 / 02 / 1998$ & 6 & 1 & 28 & 3 & 21 & 0.908 & $\mathrm{P}>0,05$ \\
\hline
\end{tabular}

$(*)=$ obtenido de promedios censales; $(* *)=$ obtenido desde máximos censales 
territoriales en el área reproductiva (Araya et al. 1987 $)^{2}$. Este índice es semejante al presentado por Boness (1991), como "operational sex ratio" (OSR) que corresponde a la razón promedio estacional de hembras fértiles por macho activo en un tiempo dado. También se determinó el índice de hembras próximas (HP) que corresponde al número de hembras próximas a un macho territorial en un perímetro de $2 \mathrm{~m}$. Este perímetro fue estimado empleando como unidad de medición la longitud de una hembra extendida en el harén evaluado, asumiendo que la hembra presenta una longitud promedio de 1,5 m (observación personal).

En tercer lugar, se determinó (c) el período de partos y cópulas. Para ello, dos observadores realizaron tres censos diarios del número de cachorros y de cópulas durante las temporadas de estudio. Los datos obtenidos fueron expresados en tablas de frecuencias a las cuales se les ajustó una ecuación logística, similar a la metodología descrita por Trites (1992) y García-Aguilar \& Aurioles-Gamboa (2003). Estas ecuaciones fueron ajustadas por medio de la suma de mínimos cuadrados determinando los distintos parámetros de ellas. Las ecuaciones empleadas fueron:

$$
P_{e}=K / 1+e^{(a-r t)}(1)
$$

donde $\mathrm{P}_{\mathrm{e}}=$ patrón estimado (parto o cópula), $\mathrm{k}$ = asintótica (partos o cópulas), $\mathrm{a}=$ constante, $\mathrm{r}$ = tasa de incremento (partos o cópulas) y $\mathrm{t}=$ tiempo.

A partir de los parámetros estimados de la ecuación (1), se determinó la frecuencia de los partos y cópulas en el tiempo (i.e., tasa de variación), graficándose el período de duración y la fecha máxima de ellos para cada temporada. La fecha máxima de pariciones fue determinada empleando la curva de frecuencia de los partos previamente diseñada, en la cual se registró el punto de mayor ocurrencia de ellas. Esta misma relación fue utilizada para

\footnotetext{
2 ARAYA H., F CONTRERAS, F CAMPOS, M ARROYO \& E RODRÍGUEZ (1987) Biología reproductiva del león marino sudamericano (Otaria flavescens) en la lobería de Punta Negra, Iquique-Chile. Anais da $2^{\text {a }}$ Reunão de Trabalho de Especialistas em Mamíferos Aquáticos da América do Sul, Rio Janeiro, Brasil: 9-16.
}

determinar la fecha del máximo de las cópulas de cada temporada.

\section{Análisis estadístico}

Los análisis estadísticos fueron realizados empleando STATISTICA 6.0 para Windows 98, 2002. Se utilizaron pruebas de G ("Loglikelihood ratio") con y sin corrección de Yates para determinar las posibles diferencias en tablas de contingencia y datos categóricos entre temporadas (e.g., índices de poliginia, proporción de animales organizada en harenes, duración de los eventos).

Debido a que el número de harenes, partos y cópulas no presentaban varianza homogénea ni una distribución normal, se aplicaron pruebas no paramétricas para compararlos entre temporadas. Se utilizaron pruebas pareadas de Wilcoxon para examinar diferencias en la cantidad de harenes, partos y cópulas entre temporadas, mediante una comparación día por día del número de ellos (i.e., compara el número total de cada evento). Se emplearon pruebas de Kolmogorov-Smirnov para examinar diferencias en el tipo de distribución de los patrones reproductivos y las formas de las curvas mediante una comparación de la frecuencia de los sucesos por día (i.e., compara las fechas de cada evento). Se aplicaron pruebas de mediana para determinar diferencias en la mediana de las fechas de los patrones reproductivos, mediante una comparación del arreglo de los datos en torno a ella (i.e., compara las fechas del máximo de cada evento) (Zar 1999, Statsoft Inc. 2001).

\section{RESULTADOS}

Harenes del sector reproductivo principal (Playa Grande)

Los harenes establecidos en Playa Grande presentaron diferencias tanto en el tamaño, número como en su distribución. Durante la temporada 1996-1997 los harenes se distribuyeron a lo largo de toda la playa. Sin embargo, durante la segunda temporada, el $70 \%$ de ellos se estableció en el lado norte de la playa, reduciendo su número y estructura, pero aumentando su densidad (i.e., 0,04 harenes $\mathrm{m}^{-2}$ en 1996-1997 y 0,12 harenes $\mathrm{m}^{-2}$ durante 1997-1998) (Fig. 3). 
(A)

(B)

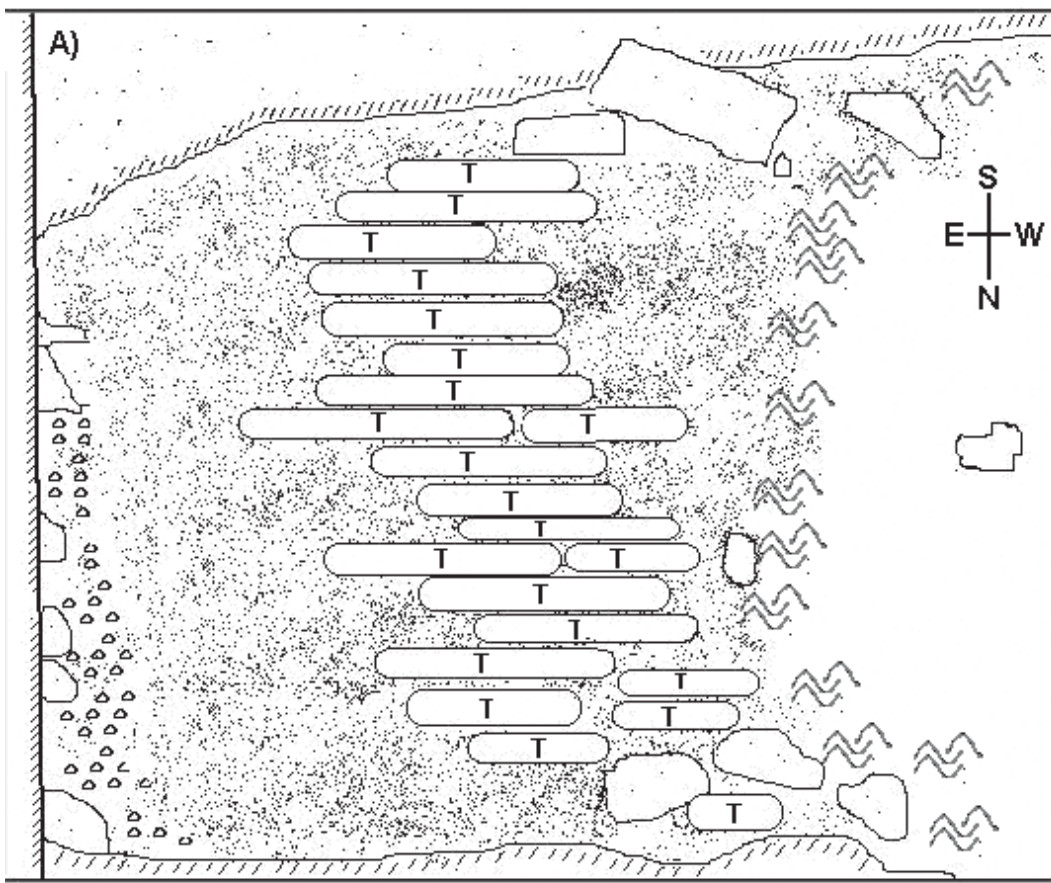

\section{Leyenda}

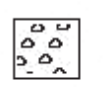

Bolones

.

Arena

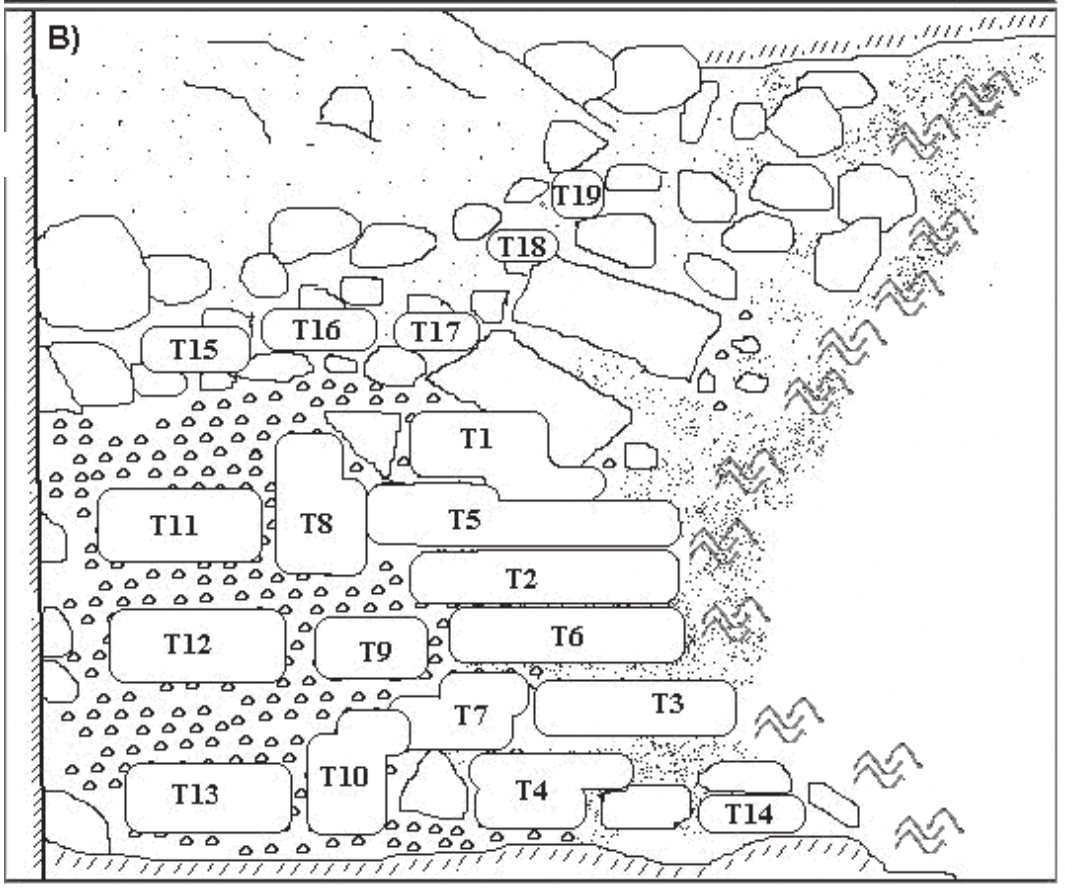

Rocas

道.

Rompiente

ij/4

Acantilado

T

Territorios

(harenes)

Fig. 3: Distribución de harenes durante las temporadas reproductivas (A) 1996-1997 y (B) 19971998, establecidos en Playa Grande, Punta Lobería, IX Región, Chile.

Spatial distribution of harems during breeding season 1996-1997 (A) and 1997-1998 (B) on Playa Grande, Punta Lobería, IXth Region, Chile. 
Fluctuación del número de harenes en Playa Grande

En la temporada 1996-1997 la formación paulatina de los harenes en Playa Grande fue difícil de monitorear, debido a que desde el inicio del muestreo (diciembre de 1996) la población era de aproximadamente 3.000 ejemplares afectando la visibilidad del proceso (Espinoza 2001). Sin embargo, se pudo identificar un mínimo de seis y un máximo de 28 harenes en esta temporada (Fig. 4).

Durante la temporada 1997-1998, el primer harén constituido fue observado en la segunda quincena de noviembre 1997, y el último, a fines de enero de 1998. La mayoría de los harenes se formaron entre el 15 y el 20 de enero de 1998, registrándose entre uno a 19 harenes. Este número fue estadísticamente menor que lo determinado durante la temporada anterior (prueba pareada de Wilcoxon, $\mathrm{T}=7,00 ; \mathrm{Z}=$ $2,690 ; \mathrm{n}=13 ; \mathrm{P}=0,007)$. Sin embargo, no se registró diferencia en la tendencia estacional de ellos (prueba de Kolmogorov-Smirnov, gl = 1; $\mathrm{P}$ $>0.1$ ) (Tabla 3).

La fecha donde se identificó el número máximo de harenes (i.e., primera semana de febrero de 1997 y 1998; prueba para la mediana, $\left.\chi^{2}=3,712 ; \mathrm{gl}=1 ; \mathrm{P}=0,054\right)$, la fecha de desintegración (i.e., 15 de febrero de
1997 y 19 de febrero de 1998) y el periodo de sincronización del $85 \%$ de ellos (i.e., entre la cuarta semana de enero y segunda semana de febrero de 1997 y 1998) no presentaron diferencias estadísticamente significativas entre temporadas (Tabla 3).

\section{Estructura poligínica de los harenes}

La proporción de macho territorial: hembra: juvenil: cachorros de los harenes fue 1:12:1:7 y 1:9:2:6 para las temporadas 1996-1997 y 1997 1998, respectivamente, sin ser estas diferencias estadísticamente significativas $(\mathrm{G}=0,449 ; \mathrm{gl}=$ 3; $\mathrm{P}>0,05)$.

Durante ambas temporadas reproductivas la cantidad de hembras y juveniles por harén presentaron tendencias similares. Se observó que durante el establecimiento y disolución de los harenes la cantidad de ellos era mayor que en el momento de sincronización. Los cachorros, por su parte, en ambas temporadas presentaron un incremento sostenido por harén hasta fines de las temporadas reproductivas. Solo se determinó diferencias estadísticas en la estructura de los harenes formados a principio de la estación reproductiva entre ambas temporadas, i.e., segunda quincena de diciembre $(\mathrm{G}=28,034$; $\mathrm{gl}=3 ; \mathrm{P}<0,05 ;$ Tabla 1).

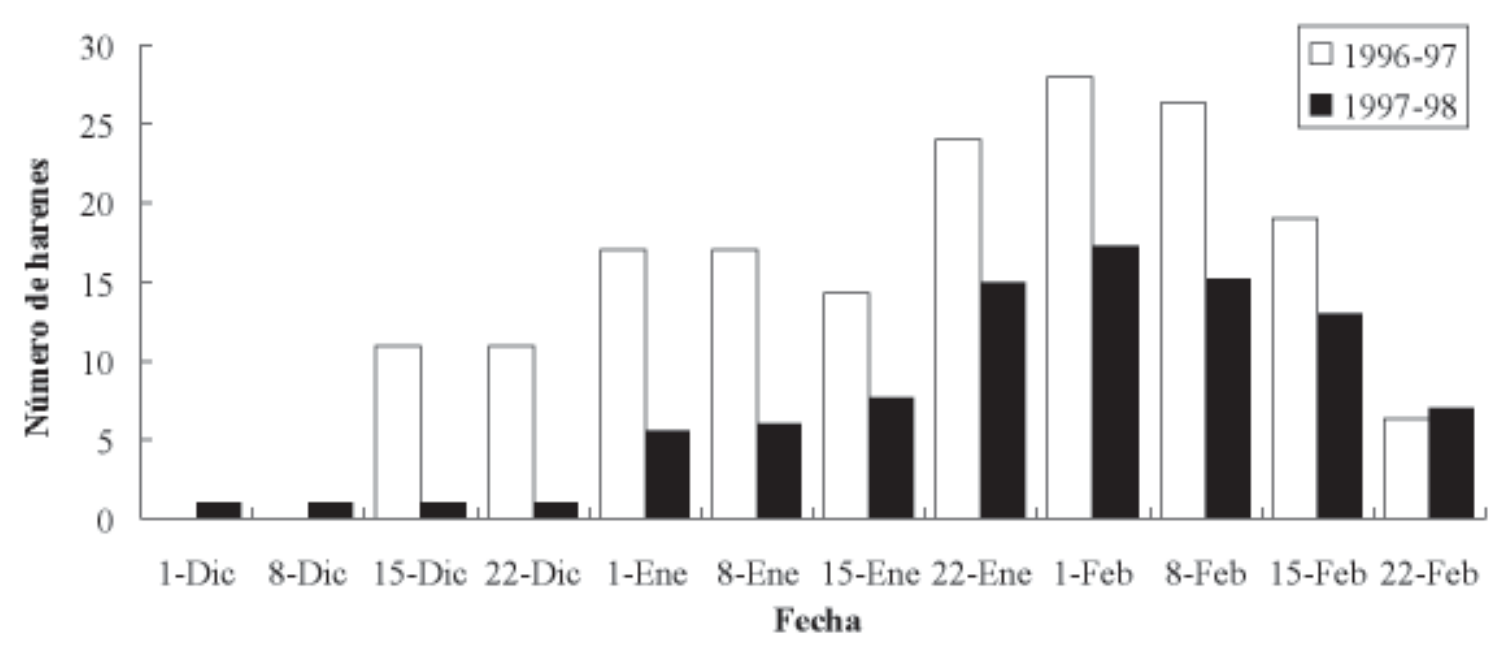

Fig. 4: Variación del número de harenes durante la temporada reproductiva 1996-1997 y 1997-1998 en la Playa Grande de Punta Lobería, IX Región de Chile.

Harem number changes during breeding season 1996-1997 and 1997-1998 on Playa Grande, Punta Lobería, IXth Region, Chile. 
TABLA 2

Niveles de poliginia determinada en la Playa Grande de la colonia reproductiva de Punta Lobería, durante las temporadas 1996-1997 y 1997-1998, IX Región. Chile. HPMT = número de hembras por macho territorial; $\mathrm{HP}=$ número de hembras próximas

Polygynous index determined for Playa Grande in a breeding sea lion colony at Punta Lobería during seasons $1996-1997$ and 1997-1998, IXth Region of Chile. HPMT = number of females per territorial male; HP = number of nearer females

\begin{tabular}{lcccccc}
\hline Temporada & Machos territoriales & Hembras* & HPMT & HP(rango) & Valor de Gc & Valor de P \\
\hline $1996-97$ & 28 & 348 & 12,4 & $11(4$ a 30) & 0.0068 & P $>0,05$ \\
$1997-98$ & 19 & 148 & 7,8 & $9(2$ a 25) & 0.0024 & $\mathrm{P}>0,05$ \\
\hline
\end{tabular}

$(*)$ = número de hembras reproductivas fue obtenido de la relación: número máximo de cachorros = hembras maduras

Al comparar el número de hembras por macho territorial (HPMT) y el número de hembras próximas (HP), los niveles de poliginia fueron semejantes (Tabla 2). Por otro lado, aunque se observa un descenso en ambos índices entre temporadas, este no fue estadísticamente significativo (HPMT: $\mathrm{G}_{\mathrm{c}}=$ 0,$645 ; \mathrm{gl}=1 ; \mathrm{P}>0,05 ; \mathrm{HP}: \mathrm{G}_{\mathrm{c}}=0,05 ; \mathrm{gl}=1 ; \mathrm{P}$ $>0,05$ ). Sin embargo, la reducción del tamaño poblacional organizado en harenes entre temporadas fue estadísticamente significativo $\left(\mathrm{G}_{\mathrm{c}}=41,56 ; \mathrm{gl}=1 ; \mathrm{P}<0,05\right)(1996-1997: 42,7$ $\%, \mathrm{n}=702, \mathrm{~N}=1644 ; 1997-1998: 32,4 \%, \mathrm{n}=$ $480, \mathrm{~N}=1482$ ).

Período de partos en el sector reproductivo principal (Playa Grande)

Durante 1996-1997 los parámetros estimados de la ecuación (1) para determinar el período de pariciones tuvieron los siguientes valores: $\mathrm{a}=$ 21,$717 ; \mathrm{r}=0,394 ; \mathrm{K}=382,2$. Durante 1997 1998 los valores de los mismos parámetros fueron: $\mathrm{a}=15,908 ; \mathrm{r}=0,294 ; \mathrm{K}=147,9$.

Al comparar el período de pariciones entre ambas temporadas de estudios, se observó que estas se desarrollaron en fechas cercanas (Tabla $3)$. Sin embargo, la cantidad promedio de partos fue estadísticamente menor en la segunda temporada (prueba de Wilcoxon, $\mathrm{T}=$ 31,$00 ; \mathrm{Z}=4,144 ; \mathrm{n}=30 ; \mathrm{P}=0,00003$ ).

Durante la primera temporada se determinó que el $89,95 \%(\mathrm{n}=344, \mathrm{~N}=383)$ de los partos fueron registrados entre el 17 al 31 de enero, es decir, en un lapso de 15 días. En la temporada siguiente este período de sincronización se registró entre el 12 y el 31 de enero, con una duración de 20 días $(89,66 \% ; \mathrm{n}=133, \mathrm{~N}=$ 148; Tabla 3). Se determinó diferencias estadísticamente significativas en la distribución de los datos entre las temporadas (prueba de Kolmogorov-Smirnov, gl = 1, $\mathrm{P}<$ 0,025) (Tabla 3). Por su parte, la fecha del máximo de pariciones durante la segunda temporada fue registrada 2 días antes que lo determinado para la primera, situación que también provocó una diferencia estadística cercana del límite de aceptación (i.e., 24 de enero de 1997 y 22 de enero de 1998; prueba para la mediana, $\left.\chi^{2}=3,905 ; \mathrm{gl}=1 ; \mathrm{P}=0,048\right)$ (Tabla 3).

Mediante los datos obtenidos se estima que la fecha media del máximo de partos en Punta Lobería correspondió al día 23 de enero $( \pm 1,71$ días).

Período de cópulas en el sector reproductivo principal (Playa Grande)

Los parámetros de la ecuación (1) empleados para determinar el período de cópulas durante la primera temporada fueron los siguientes: $\mathrm{a}=$ 14,$012 ; r=0,230 ; K=117$. Durante la segunda temporada los valores de estos parámetros fueron: $\mathrm{a}=15,296 ; \mathrm{r}=0,241 ; \mathrm{K}=147,6$.

El período de cópulas entre ambas temporadas se presentó en fechas cercanas sin ser estas estadísticamente diferentes (prueba de Kolmogorov-Smirnov, $\mathrm{gl}=1, \mathrm{P}<0,1)$, al igual que lo registrado con las pariciones (Tabla 3 ). Sin embargo, se registró diferencias estadísticas en el número de cópulas entre las temporadas (prueba de Wilcoxon, $\mathrm{T}=165,00 ; \mathrm{Z}=2,813 ; \mathrm{n}$ $=37 ; \mathrm{P}=0,004)($ Tabla 3$)$. 
TABLA 3

Fecha de ocurrencia de los patrones reproductivos del lobo marino común durante dos temporadas consecutivas (Punta Lobería, IX Región-Chile). Se entrega la diferencia en días, porcentaje de variación y valores de Gc

Reproductive patterns dates in the sea lions breeding colony (Punta Lobería, IXth Region-Chile). It is indicated the difference in days, the variation percentage and Gc values

\begin{tabular}{|c|c|c|c|c|c|}
\hline Evento & 1996-1997 & 1997-1998 & Desfase (días) & $\begin{array}{c}\text { Variación } \\
\text { porcentual }(\%)^{\mathrm{a}}\end{array}$ & Valor de Gc y P \\
\hline Primer harén formado & Sin datos & $\begin{array}{l}\text { Cuarta semana } \\
\text { de noviembre }\end{array}$ & - & - & - \\
\hline Fecha de desintegración & $\begin{array}{l}\text { Tercera semana } \\
\text { de febrero }\end{array}$ & $\begin{array}{l}\text { Tercera semana } \\
\text { de febrero }\end{array}$ & 0 & 0 & - \\
\hline Fecha del máximo & $\begin{array}{l}\text { Primera semana } \\
\text { de febrero }\end{array}$ & $\begin{array}{l}\text { Primera semana } \\
\text { de febrero }\end{array}$ & 0 & 0 & $\mathrm{P}>0,05$ \\
\hline $\begin{array}{l}\text { Permanecia del } 85 \% \\
\text { de los harenes (días) }\end{array}$ & $\begin{array}{l}\text { Cuarta semana de } \\
\text { enero a segunda semana } \\
\text { de febrero }(21)\end{array}$ & $\begin{array}{l}\text { Cuarta semana de } \\
\text { enero a segunda semana } \\
\text { de febrero }(21)\end{array}$ & a & 0 & $P>0,05$ \\
\hline Primer parto observado & 21 de diciembre & 6 de enero & 16 & 76 & $3,923^{*}$ \\
\hline Último parto observado & 22 de febrero & 24 de febrero & 2 & 9 & 0,021 \\
\hline Fecha máximo de partos & 24 de enero & 22 de enero & -2 & -8 & $\mathrm{P}<0,05$ \\
\hline $\begin{array}{l}\text { Sincronización de las } \\
\text { pariciones ( } 90 \% \text { en días) }\end{array}$ & 17 al 31 de enero (15) & 12 al 31 de enero (20) & $-5(5)$ & $-29(33)$ & $\mathrm{P}<0,05(0,458)$ \\
\hline Primera cópula observada & 2 de enero & 22 de enero & 20 & 1000 & $17,232 *$ \\
\hline Última cópula observada & 22 de febrero & 23 de febrero & 1 & 4 & 0,000 \\
\hline Fecha máximo de cópulas & 29 de enero & 1 de febrero & 3 & 10 & $\mathrm{P}<0,05$ \\
\hline $\begin{array}{l}\text { Sincronización de las } \\
\text { cópulas ( } 90 \% \text { en días) }\end{array}$ & $\begin{array}{l}16 \text { de enero al } \\
10 \text { de febrero }(26)\end{array}$ & $\begin{array}{l}20 \text { de enero al } \\
13 \text { de febrero }(25)\end{array}$ & $4(-1)$ & $25(-4)$ & $\mathrm{P}<0,05(0,000)$ \\
\hline
\end{tabular}

$(*)=$ marca diferencia estadísticamente significativa; $\mathrm{G}_{\mathrm{c}}=3,841 ; \alpha=0,05, \mathrm{gl}=1 ; \mathrm{P}<0,05$;

$\left({ }^{a}\right)=$ se entrega el porcentaje de variación entre temporadas, signo negativo indica la existencia de una disminución, signo positivo hace referencia a un incremento

El período de sincronización de las cópulas tuvo una diferencia de 1 día entre temporadas reproductivas, sin ser ello estadísticamente diferente (Tabla 3). Durante la primera temporada el 90,2 \% de las cópulas $(\mathrm{n}=106, \mathrm{~N}$ $=117$ ) fueron registradas entre el 16 enero al 10 de febrero, i.e., en 26 días. En la segunda temporada el $90,6 \%$ de ellas $(\mathrm{n}=134, \mathrm{~N}=148)$ fueron identificadas entre el 20 de enero y el 13 febrero de 1998, i.e., en 25 días. Por su parte, la fecha del máximo de cópulas presentó un desfase de 3 días entre temporadas (i.e., 29 de enero de 1997 y 1 febrero de 1998), siendo esta diferencia estadísticamente significativa (prueba para la mediana, $\chi^{2}=6,129 ; \mathrm{gl}=1 ; \mathrm{P}=0,013$ ).

Mediante el método logístico se estimó que la fecha media del máximo de cópulas en Punta Lobería fue el día 31 de enero $( \pm 2,12$ días $)$.

La diferencia entre la fecha del máximo de pariciones y del máximo de cópulas permite determinar el inicio del período de estro. De esta forma, durante la primera temporada las hembras parteras fueron copuladas 5 días después del parto, período inferior al determinado para la segunda temporada (i.e., 10 días posparto). Esta variación no fue estadísticamente significativa $\left(\mathrm{G}_{\mathrm{c}}=1,07 ; \mathrm{gl}=\right.$ $1 ; \mathrm{P}>0,05)$. 


\section{DISCUSIÓN}

En general se asume que en las colonias reproductivas de lobos marinos, el número, tamaño y ubicación de los harenes se mantiene estable durante temporadas reproductivas sucesivas, aspecto apoyado por una variada literatura (Araya et al. 1986, 1987, Trites 1992, Acevedo 1999, Guzmán 2002, Acevedo et al. 2003, García-Aguilar \& Aurioles-Gamboa 2003). Sin embargo, estas tres características difirieron en la Playa Grande de Punta Lobería junto con otros aspectos organizacionales de la lobera (e.g., índice de poliginia, estructura etarias y el número de ejemplares organizados en harenes).

La existencia de estas modificaciones se ha relacionado con el efecto de un derrumbe de rocas y bolones de arenisca ocurrido en julio de 1997. En esta oportunidad, el nuevo sustrato incorporado alteró la topografía del sector y las características térmicas del sustrato. Ambas situaciones provocaron tanto una reducción del espacio óptimo para el establecimiento de los lobos marinos como modificaciones en las conductas termorregulatorias de los animales. En este sentido, se ha podido determinar una relación entre tipos de conductas sociales, reproductivas y termorregulatorias con las características topográficas y térmicas de los territorios donde se establecen los lobos marinos y los "harenes" (Campagna \& Le Boeuf 1988, Cassini \& Vila 1990, Pavés 2001). Los derrumbes no solo fueron registrados en invierno, sino también durante las temporadas estivales donde se observaron modificaciones en la conducta reproductiva, sistema de apareamiento y estrategia reproductiva de los machos territoriales en la playa reproductiva, conjuntamente con continuas desintegración de los harenes cada vez que se producían las estampidas (Pavés 2001, Pavés et al. 2002a ${ }^{3}$, $\left.2002 b^{4}\right)$

\footnotetext{
3 PAVÉS H, J OPORTO, R SCHLATTER \& AE CROVETTO (2002a) Variación de la conducta social del lobo marino común (Otaria flavescens, Shaw 1800) en Punta Lobería, IX región. XXII Congreso de Ciencias del Mar, Valdivia, Chile, 28 al 31 de mayo: 39.

4 PAVÉS H, C ESPINOZA \& R SCHLATTER (2002b) Variaciones en los patrones conductuales reproductivos del lobo marino común (Otaria flavescens, Shaw 1800) en Punta Lobería, IX Región. Décima RT y Cuarto Congreso SOLAMAC, Valdivia, Chile, 14 al 19 de octubre: 44.
}

Aunque se registraron estas importantes perturbaciones en el sistema, conductas y eventos reproductivos en Punta Lobería se mantuvieron estables, i.e., estacionalidad de los harenes y el período de sincronización de las pariciones y de las cópulas. Estos tres eventos presentaron ciertas diferencias entre temporadas, sin embargo, fueron registrados durante el mismo período de tiempo, condición que típicamente se observa en la mayoría de los Otariidae (Campagna 1985, Boness 1991, Majluf 1992, Trites 1992, García-Aguilar \& AuriolesGamboa 2003). La diferencia registrada en la fecha del máximo de las cópulas en Punta Lobería se asocia al adelantamiento o retraso de la fecha del máximo de los partos entre temporadas, situación también identificada en otras colonias (Campagna 1985, Goldsworthy \& Shaughenessy 1994). A su vez, la alteración en la fecha de las pariciones registrado en Punta Lobería puede ser explicada por la presencia de hembras de mayor edad o hembras inmigrantes que llegan a parir primero, alterando la fecha del máximo año tras año (Trites 1992).

La estabilidad en los eventos o "patrones reproductivos" en Punta Lobería estaría relacionada con la rigidez del ciclo reproductivo de $O$. flavescens en el sector. Este ciclo tendría una duración aproximada de 11 meses y 24 días calculados entre el máximo de cópulas y el máximo de pariciones. Esta información permitiría precisar una diapausa embrionaria de dos meses 24 días, considerando los 9 meses restantes empleados en la gestación embrionaria, según lo indicado por Costa (1991). La duración de este ciclo reproductivo, que en definitiva determinaría la estacionalidad reproductiva, está asociada a efectos de presión de selección natural sobre la sobrevivencia de los progenitores y la descendencia (Testa 2002). Los factores que podrían estar generando está presión de selección natural tienen relación con una marcada estacionalidad climática identificada en la colonia. Esta estacionalidad provocaría la presencia de condiciones climáticas favorables y la disponibilidad de abundantes recursos alimentarios en el medio durante un período discreto de tiempo (Riedman 1990, Boness 1991, Majluf 1992, Trites \& Antonelis 1994, Soto et al. 2004).

En ambientes donde no se registra una marcada estacionalidad climática y existe una 
reducida oferta alimentaria durante todo el año, el período reproductivo es asincrónico y no estacional (e.g., Australia, Costa \& Gales 2003). Es así como el lobo marino australiano (Neophoca cinerea) presenta un ciclo reproductivo de 18 meses, pariendo a través de todo el año (Higgins 1993), situación que contrasta con lo registrado en $O$. flavescens de Punta Lobería.

Al comparar la fecha de desarrollo de los tres principales eventos reproductivos obtenidos en Punta Lobería con los de otras localidades, es posible observar diferencias entre ellos. Es así como el período de formación de los harenes, el período de sincronización de los partos y cópulas de $O$. flavescens en el norte de Chile, se registró en fechas anteriores, presentando a su vez una mayor duración que aquellas registradas en el centro sur de Chile (Araya et al. 1986, Acevedo 1999, Acevedo et al. 2003). En Punta Lobería se constató que el $90 \%$ de los partos sucede en 18 días en promedio, período menor a los 31 días determinados en Punta Negra, Iquique (Araya et al. 1986, 1987. Pavés et al., resultados no publicados). Por su parte, el $90 \%$ de las cópulas se registró en un promedio de 26 días, menor a lo estimado para Iquique (i.e., 44 días, Araya et al. 1986, Acevedo 1999, Pavés et al., resultados no publicados), pero mayor a lo registrado por Campagna (1985) en el sur de Argentina (11 días, 1985).

La existencia de un efecto latitudinal sobre la sincronización reproductiva también ha sido identificado en otras especies de otáridos que presentan una amplia área de distribución geográfica (e.g., Zalophus californianus, García-Aguilar \& Aurioles-Gamboa 2003). Situación que, según Boness (1991), evidencia una fuerte relación entre las condiciones ambientales locales y la biología básica de la especie. Boness (1991) logra determinar una relación inversa entre latitud y la sincronización reproductiva tanto a nivel interespecífico como intraespecífico. Es así como aquellas especies/poblaciones establecidas a menor latitud presentan un período de sincronización de mayor duración. Como Boness (1991) comenta, esta relación estaría determinada por el efecto de la estacionalidad climática y de la disponibilidad de recursos alimentarios sobre la biología de las especies, relación que ha podido ser determinada en algunas especies de otáridos (e.g., Arctocephalus australis: Majluf 1992, Soto et al. 2004; Callorinchus ursinus: Trites \& Antonelis 1994).

En este estudio se pudo determinar que aunque las conductas sociales organizacionales y reproductivas (e.g., proporciones etarias, número de harenes, densidad de los harenes, índice de poliginia) pueden variar de intensidad y localización por el efecto de factores perturbadores, los patrones reproductivos en el interior del harén (i.e., partos y cópulas) se registran en el mismo período de tiempo entre temporadas necesitando el efecto de perturbaciones ambientales de mayor duración o intensidad para ser alterados (Sielfeld \& Guzmán 2002, Soto et al. 2004, Tabla 3).

\section{AGRADECIMIENTOS}

A Jorge Oporto y la Corporación Terra Australis-Valdivia que financiaron y apoyaron el desarrollo de esta investigación. A Rodrigo Hucke-Gaete, Roberto Nespolo y dos revisores anónimos por sus comentarios al manuscrito. A Jorge Acevedo (Universidad Arturo PratInstituto Antártico Chileno), Jaquelin Bianco (Universidad de la Republica Oriental del Uruguay), Claudio Campagna (Centro Nacional Patagónico), Luis Cappozzo (Museo Argentino de Ciencias Naturales), Jeremy David (Marine and Coastal Management- South Africa), Carlos Guerra (Universidad de Antofagasta), Sonja Heinrich (Universitat St Andrews), Burney Le Boeuf (Univeristy of California, Santa Cruz), Nathan McNally (University of Otago), Maritza Sepúlveda (Universidad de Valparaíso), Peter Shaughnessy (Commenwealth Scientific and Industrial Research Organization), Walter Sielfeld (Universidad Arturo Prat), Andrew Trites (University of British Columbia), Anne York (National Oceanic Atmospheric Administration) por la literatura facilitada. A la comunidad de Punta Lobería, Pilolcura y Coicoi por la ayuda prestada, gracias. Héctor Pavés es estudiante del Programa de Doctorado en Ciencias y fue apoyado por una beca de MECE Educación Superior (AUS 0111) y por la Universidad Austral de Chile, Chile. 


\section{LITERATURA CITADA}

ACEVEDO J (1999) Temporada de reproducción del lobo marino común (Otaria flavescens, Shaw 1800), en la lobera de Punta Negra $\left(20^{\circ} 50^{\prime}\right.$ S. $)$, Iquique, durante las temporadas 1995/96 y 1996/97: crías. Tesis de Título, Facultad de Ciencias, Universidad Arturo Prat, Iquique, Chile. viii +170 pp.

ACEVEDO J, AGUAYO-LOBO A \& W SIELFELD (2003) Eventos reproductivos del león marino común Otaria flavescens (Shaw 1800), en el norte de Chile (Pacífico suroriental). Revista de Biología Marina y Oceanografía (Chile) 38: 69-75

ARAYA H, ARROYO M, CAMPOS F \& F CONTRERAS (1986) Conducta reproductiva del lobo marino común (Otaria flavescens) Iquique-Chile. 19841985. Memoria para optar al título de Profesor de Estado en Biología y Ciencias, Universidad Arturo Prat, Iquique, Chile. ix +165 pp.

BONESS D (1991) Determinants of mating system in the Otariidae (Pinipedia). En: Renouf D (ed) Behaviour of pinniipeds: 1-35. Chappman and Hall Ltd., New York, New York, USA.

BOWEN W (1991) Behavioural ecology of pinniped neonates. En: Renouf D (ed) Behaviour of pinniipeds: 66-128. Chapman and Hall Ltd., New York, New York, USA.

CAMPAGNA C (1985) The breeding cycle of the southern sea lion (Otaria byronia). Marine Mammal Science 1: $210-218$

CAMPAGNA C \& B LE BOEUF (1988) Thermoregulatory behaviour of southern sea lions and its effect on mating strategies. Behaviour 107: 72-90.

CAMPAGNA C, LEWIS M \& R BALDI (1993) Breeding biology of southern elephant seals in Patagonia. Marine Mammal Science 9: 34-37.

CAPPOZZO L \& M JUNIN (1991) Estado de conservación de los mamíferos marinos del Atlántico sudoccidental. Informes y Estudios del Programa de Mares Regionales del PNUMA, Número 138. 250 pp.

CASSINI M \& B VILA (1990) Male mating behaviour of the southern sea lions. Bulletin of Marine Science 46: 555-559.

COSTA D (1991) Reproductive and foranging energetics of pinnipeds: Implications for life history patterns. En: Renouf D (ed) Behaviour of pinniipeds: 300344. Chappman and Hall Ltd., New York, New York, USA

COSTA D \& N GALES (2003) Energetics of a benthic diver: seasonal foraging ecology of the Australian sea lion, Neophoca cinerea. Ecological Monographs 73: 27-43.

ESPINOZA C (2001) Dinámica poblacional del lobo marino común sudamericano (Otaria flavescens, Shaw 1800) en la colonia de Punta Lobería, IX Región de Chile. Tesis de Licenciatura, Facultad de Ciencias, Universidad Austral de Chile, Valdivia, Chile. xxiii $+150 \mathrm{pp}$.

FAO (1993) FAO species identification guide for marine mammals of the world. Otariidae Eared Seals. 232 pp.

GARCÍA-AGUILAR M \& D AURIOLES-GAMBOA (2003) Breeding season of the California sea lion (Zalophus californianus) in Gulf of California, México. Aquatic Mammals 29: 67-76.
GOLDSWORTHY S \& P SHAUGHNESSY (1994) Breeding biology and haul-out pattern of the New Zealand fur seal, Arctocephalus forsteri, at Cape Gantheaume, South Australia. Wildlife Research 21: 365-376.

GUZMÁN A (2002) Temporada reproductiva 1998-99/ 1999-2000 del lobo marino común, Otaria flavescens (Shaw 1800), en la lobera de Punta Negra (2050'S), posterior al evento El Niño 1997/ 1998. Tesis para optar al Título de Biólogo Marino, Universidad Arturo Prat, Iquique, Chile. v + 55 pp.

HIGGINS L (1993) The nonanual, nonseasonal breeding cycle of the Australian sea lion, Neophoca cinerea. Journal of Mammalogy 74: 270-274.

MAJLUF P (1992) Timing of births and juvenile mortality in South American fur seal in Perú. Journal of Zoology, London 227: 367-383.

PAVÉS H (2001) Variación de la conducta social reproductiva del lobo marino común (Otaria flavescens, Shaw 1800) en Punta Lobería, IX Región (1996-97 y 1997-98). Tesis de Licenciatura, Facultad de Ciencias, Universidad Austral de Chile, Valdivia, Chile. xxii +203 pp

RIEDMAN M (1990) The pinnipeds: seals, sealions, and walruses. University of California Press, Berkeley, California, USA. xxiii + 439 pp.

RIVERA A (1990) Etología: displays agonísticos presentes en Otaria flavescens (Shaw, 1800) Punta Hualpén, Chile (Mammalia: Otariidae). Gayana Zoología (Chile) 54: 33-49

SEPÚLVEDA M (1999) Circaritmos de actividad del lobo marino común Otaria flavescens (Carnívora: Otariidae) y su relación con la salmonicultura en la $\mathrm{X}$ Región, Chile. Tesis para optar al Título Profesional de Biólogo Marino, Universidad de Valparaíso, Valparaíso, Chile. 130 pp.

SIELFELD W (1983) Mamíferos marinos de Chile. Ediciones Universidad de Chile, Santiago, Chile. $199 \mathrm{pp}$.

SIELFELD W (1999) Estado del conocimiento sobre conservación y preservación de Otaria flavescens (Shaw 1800) y Arctocephalus australis (Zimmerman 1783) en las costas de Chile. Estudios Oceanológicos (Chile) 18: 81-96.

SIELFELD W \& A GUZMÁN (2002) Effect of El Niño $1997 / 98$ on a population of the southern sea lion (Otaria flavescens Shaw) from Punta Patache/Punta Negra (Iquique, Chile). Investigaciones Marinas (Chile) 30: 157-160.

SOTO K, A TRITES \& M ARIAS-SCHREIBER (2004) The effects of prey availability on pup mortality and the timing of birth of South American sea lions (Otaria flavescens) in Perú. Journal of Zoology, London 264: 419-428.

STATSOFT INC (2001) Statistica (data análisis software system, Versión 8. www.statsoft.com).

TESTA J (2002) Does predation on neonates inherently select for earlier birth? Journal of Mammalogy 83: 699-706.

TRITES A (1992) Reproductive synchrony and the estimation of mean date of birth from daily counts of Northern fur seal pups. Marine Mammal Science 8: 44-56.

TRITES A \& G ANTONELIS (1994) The influence of climatic seasonality on the life cycle of the Pribilof northern fur seal. Marine Mammal Science 10: 311324. 
VAZ-FERRERIA. R (1965) Ecología terrestre y marina de los pinnipedios del Atlántico sudoccidental. Anais da Academia Brasileira de Ciencias (Brasil), Suplemento 2, 137: 179-191.

Vaz-Ferreira R (1982) Otaria flavescens (Shaw), South American Sea Lion. In: FAO Fisheries Series 5 (eds) Mammals in the Seas: Small cetaceans, seals, sirenias and otters. 4: 477-495.

ZAR J (1999) Biostatistical analysis. Fourth edition. Prentice-Hall, Upper Saddle River, New Jersey, USA. 860 pp.

Editor Asociado: Mauricio Lima

Recibido el 19 de agosto de 2004; aceptado el 22 de junio de 2005 